\title{
Subarachnoid Hemorrhage from Posterior Cerebral Artery Aneurysm during Puerperium - Case Report and Review of Literature
}

\author{
Karl-Michael Schebesch ${ }^{1 *}$, Petra Schödel ${ }^{1}$, Janine Rennert ${ }^{2}$, Karl-Heinz Mark ${ }^{3}$ and Alexander Brawanski ${ }^{1}$
}

${ }^{1}$ Department of Neurosurgery, Medical Center, University of Regensburg, Germany

${ }^{2}$ Department of Neuroradiology, Medical Center, University of Regensburg, Germany

${ }^{3}$ Department of Obstetrics and Gynecology, Medical Center, Weiden, Germany

\begin{abstract}
Subarachnoid hemorrhages (SAH) due to true aneurysms of the Posterior Cerebral Artery (PCA) during puerperium in young and healthy females are extremely rare.

We present the case of a 31-year old, healthy woman that experienced a spontaneous SAH due to a PCA aneurysm, arising from the P3 segment, 9 days post-delivery. The aneurysm was successfully treated via an endovascular approach and the patient recovered well. After 21 days she was discharged from hospital without neurological deficits. The clinical course is described in detail and illustrated with a computed tomography scan (CT) and digital subtraction angiography (DSA) pre - and post-embolization.
\end{abstract}

The literature is reviewed and possible etiologies of the formation and rupture of the aneurysm are discussed.

Keywords: Subarachnoid hemorrhage; Puerperium; Posterior cerebral artery aneurysm

\section{Introduction}

Circulatory disorders, cerebrovascular diseases and hemorrhagic stroke are associated with pregnancy, delivery and puerperium [1-3]. In the Western world, maternal aneurismal subarachnoid hemorrhage (SAH) occurs in $8-31$ per 100,000 deliveries [4] and accounts for up to $10 \%$ of maternal deaths in pregnant women [5]. In an epidemiological survey Jung et al. [1] investigated a history of hypertension and diabetes, a family history of stroke, smoking status and alcohol consumption as potential confounders of hemorrhagic stroke in 471 women with parity. Particularly pregnant women, suffering from preeclampsia-eclampsia or cardiomyopathy are at high risk [3,6,7]. Some authors suggested transient degeneration and turnover of the vessel wall - presumably based on hormonal changes - in combination with postpartum or pregnancy-related hypertension as potential causes of aneurysm formation and rupture $[1,2,4,6,7]$.

Due to our knowledge, spontaneous SAH due to an aneurysm of the quadrigeminal (P3-) segment of the Posterior Cerebral Artery (PCA) during puerperium has not been reported yet. Inoue et al. [8] reported one case of $\mathrm{SAH}$ postpartum due to a dissecting aneurysm of the PCA in a young and healthy Asian woman that fully recovered [8] and Groenestege et al. [4] described the rupture of a PCA aneurysm in a 32-year old healthy woman during the late-pregnancy, resulting in a fatal outcome.

We report on a healthy woman that experienced a spontaneous $\mathrm{SAH}$ due to an aneurysm of the P3 segment of the PCA during puerperium, 9 days post-delivery. During pregnancy, she did not suffer from preeclampsia-eclampsia, diabetes or hypertension. This case is noteworthy for the unusual time course of the aneurismal rupture and the rare location of the aneurismal malformation.

\section{Case Report}

A healthy, 31-year old female (p1, g2) was admitted to the neurosurgical department after uncomplicated vaginal delivery of a healthy child 9 days ago. During pregnancy she did not suffer from preeclampsia-eclampsia, diabetes or hypertension. Merely during the prolonged interval of delivery (14-15 hours) she experienced elevated blood pressures of up to $180 / 95 \mathrm{mmHg}$. She was discharged from the hospital without complications on day 4 post-delivery. Five days later, she presented disoriented with heavy cephalgia and strong neck pain to our emergency unit. The initial CT scan revealed a moderate SAH with smooth layers of blood intraventricularily (Fisher grading II) and an acute hydrocephalus (Figure 1). The contrast enhanced CT-scan showed a vascular malformation of the left posterior cerebral artery (PCA), strongly suspicious for an arterial aneurysm.

Under sedation, a ventricular tube was inserted via a burrhole trephine right frontally and the patient was transferred to the angiography suite. A 3-dimensional digital subtraction angiography (DSA) depicted a single, regular shaped aneurysm of the PCA in the P3 segment with a diameter of approx. 3 x $3 \mathrm{~mm}$ (Figure 2). Subsequently, the aneurysm was completely embolized using 3 helical coils altogether (Figure 3).

The patient awoke promptly and showed no new neurological deficit. For the next 12 days, she was kept on the neurosurgical intensive care unit (ICU) and was treated according to our standard ICU protocol for SAH. The intracranial pressure (ICP) was continuously monitored via the ventricular tube for 12 days and transcranial Doppler Sonography (TCD) was performed daily. The mean velocities of the main arteries of the anterior circulation increased up to $180 \mathrm{~cm} / \mathrm{sec}$ (left Middle

*Corresponding author: Karl-Michael Schebesch, Department of Neurosurgery, Medical Center of the University of Regensburg, Franz-Josef-Strauss Allee 11, 93053 Regensburg, Germany, Tel: ++49 +941944 9001; Fax: ++49 +941944 9002; E-mail: karl-michael.schebesch@klinik.uni-regensburg.de

Received February 21, 2012; Accepted March 06, 2012; Published March 12 2012

Citation: Schebesch KM, Schödel P, Rennert J, Mark KH, Brawanski A (2012) Subarachnoid Hemorrhage from Posterior Cerebral Artery Aneurysm during Puerperium - Case Report and Review of Literature. J Neurol Neurophysiol 3:124. doi:10.4172/2155-9562.1000124

Copyright: ( 2012 Schebesch KM, et al. This is an open-access article distributed under the terms of the Creative Commons Attribution License, which permits unrestricted use, distribution, and reproduction in any medium, provided the original author and source are credited. 


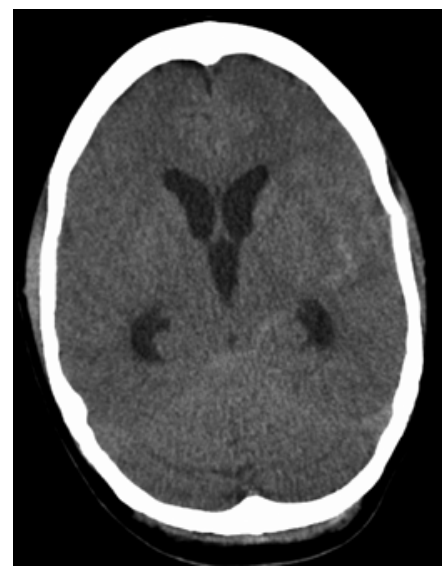

Figure 1: Initial CT scan obtaining the SAH in the fourth ventricle and in the Cisterna insularis.

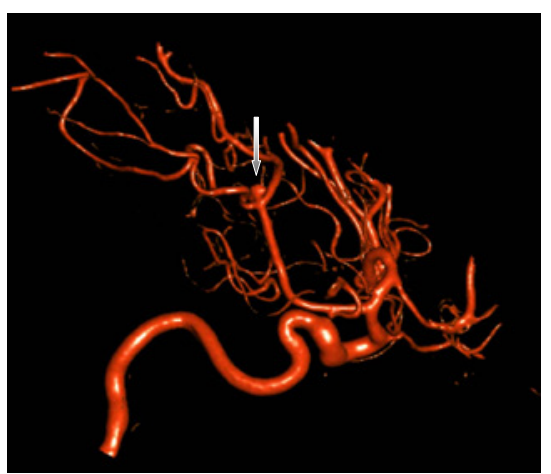

Figure 2: 3D rotational angiography revealing the regular shaped aneurysm of the PCA in the P3 segment (grey arrow).

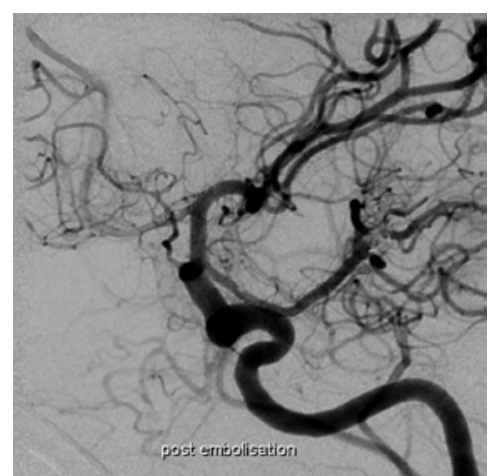

Figure 3: Angiographical control of the embolized aneurysm.

Cerebral Artery, MCA) but a delayed ischemic deficit (DCI) was ruled out clinically. Daily gynecological evaluation was unremarkable.

On day 12 after the initial ictus, the external ventricular tube was removed and the patient was transferred from the ICU to the neurosurgical general floor. After 21 days the patient was discharged from hospital without neurological deficits.

\section{Discussion}

More than $50 \%$ of ruptured arterial aneurysms in females below the age of 40 years are pregnancy-related [9]. Admittedly, in their analysis of the Utrecht population $(n=244)$ Groenestege et al. [4] found no increased risk for SAH during pregnancy, delivery and puerperium but in a nationwide epidemiological overview, de Swiet [5] correlated approx. $10 \%$ of all maternal deaths in the UK with spontaneous SAH [5] and Barno and Freeman [10] found $4.4 \%$ of all maternal deaths between 1950 and 1973 in Minnesota directly linked to SAH. Some authors emphasized an increased risk of stroke and SAH during the first six weeks post-delivery $[2,6]$, particularly concerning women with peripartum cerebral venous thrombosis and preeclampsia-eclampsia $[3,7]$.

\section{Risk factors}

The presented 31-year old woman had no history of hypertension, diabetes or drug - and alcohol consumption, she did not smoke and never had symptoms suggestive of preeclampsia-eclampsia during pregnancy.

The etiology of her peripartum SAH remains unknown. A multicenter case-control study was presented by Jung et al. [1], including 471 female cases (women with parity) with hemorrhagic stroke. It could be shown that an increased number of childbirths may be related to an increased risk for SAH and intracerebral hemorrhage [11]. Some cases were reported that identified specific cerebrovascular disorders as Moyamoya or postpartum cerebral angiopathy [12] triggering peripartum SAH, but these reports are extremely rare. Barrett et al. [9] presumed that hemodynamic and endocrine changes of pregnancy may result in arterial wall alterations, potentially leading to aneurysm formation and - rupture. However, many of the maternal SAHpatients were healthy and had no history of preeclampsia-eclampsia $[1,2,4-7,10,13]$. Transient vessel degeneration and a decreased ability in compensating hemodynamic changes are conceivably enhanced by hormonal alterations, as suggested by Barrett et al. [9]. Although the effective risk remains marginal, spontaneous SAH due to aneurysmal rupture potentially concerns all pregnant women.

\section{Localization of the aneurysm}

The aneurysm was depicted angiographically in the left quadrigeminal P3 segment (quadrigeminal and geniculate branches). It was a non-dissecting, saccular, regular shaped aneurysm with a diameter of $3 \mathrm{~mm}$. The aneurysm was embolized using helical coils without any complications.

Aneurysms of the PCA, especially of the P3 segment, are very rare [14]. Inoue et al. [8] reported a case of a dissecting aneurysm of the P3 segment of the PCA in a 30-year old, healthy woman that experienced a SAH six days post-delivery. In this publication, the literature was reviewed for dissecting aneurysms of the PCA and 40 cases were presented. 26 patients were younger than 40 years and $7 \mathrm{had}$ an aneurysm arising from the $\mathrm{P} 3$ segment. Honda et al. [15] presented a case series of 10 patients with true PCA aneurysms that were treated surgically. Only one patient had an aneurysm of the P3 segment. The development of aneurysms in such peripheral localization is difficult to explain. The intraluminal wall pressure, the pulsatile hemodynamic variations and the blood flow velocities are moderate and impingement points, where the blood flow meets the vessel wall exactly perpendicularly, are rarely present. It may be assumed that preexisting damage of endothelium and smooth muscle layers promoted the formation of the aneurysm, but the time point of the rupture, 9 days post-delivery, remains obscure.

The missing history of any disorder or disease, related or unrelated to parity, pregnancy or delivery in this 31-year old woman, strongly 
supports the assumption that hormonal changes induced the formation and the rupture of the aneurysm.

\section{Conclusion}

Despite the relatively low overall-risk in young and healthy women for SAH due to intracerebral aneurysms during pregnancy, delivery and puerperium, one should have a high degree of suspicion in any case of neurological deterioration. Immediate radiological diagnosis of the SAH and of the aneurysm is mandatory and early neurosurgical/ neuroradiological intervention should be achieved.

\section{References}

1. Jung SY, Bae HJ, Park BJ, Yoon BW (2010) Parity and risk of hemorrhagid strokes. Neurology 74: 1424-1429,

2. Salonen Ros H, Lichtenstein P, Bellocco R, Petersson G, Cnattingius S (2001 Increased risks of circulatory diseases in late pregnancy and puerperium. Epidemiology 12: 456-460,

3. Sloan MA, Stern BJ (2003) Cerebrovascular Disease in Pregnancy. Curr Treat Dptions Neurol 5: 391-407.

4. Tiel Groenestege AT, Rinkel GJ, van der Bom JG, Algra A, Klijn CJ (2009) The risk of aneurysmal subarachnoid hemorrhage during pregnancy, delivery and the puerperium in the Utrecht population: case-crossover study and standardized incidence ratio estimation. Stroke 40: 1148-1151.

5. de Swiet M (2000) Maternal mortality: confidential enquiries into materna deaths in the United Kingdom. Am J Obstet Gynecol 182: 760-766.
6. Helms AK, Kittner SJ (2005) Pregnancy and stroke. CNS Spectr 10: 580-587.

7. Jeng JS, Tang SC, Yip PK (2004) Stroke in women of reproductive age comparison between stroke related and unrelated to pregnancy. J Neurol Sc 21: 25-29,

8. noue T, Nishimura S, Hayashi N, Numagami Y, Takazawa H, et al. (2007 Postpartum dissecting aneurysm of the posterior cerebral artery. J Clin Neurosci 14: 576-581

9. Barrett JM, Van Hooydonk JE, Boehm FH (1982) Pregnancy-related rupture of arterial aneurysms. Obstet Gynecol Surv 37: 557-566.

10. Barno A, Freeman DW (1976) Maternal deaths due to spontaneous Subarachnoid hemorrhage. Am J Obstet Gynecol 125: 384-392.

11. Matsumoto Y, Asada M, Mukubou M (2009) Postpartum subarachnoid hemorrhage due to Moyamoya disease associated with renal artery stenosis. Dbstet Gynaecol Res 35: 787-789,

12. Sengoku R, Iguchi $Y$, Yaguchi $H$, Sato $H$, Inoue $K$ (2005) [A case of postpartum cerebral angiopathy with intracranial hemorrhage and subarachnoid hemorrhage immediately after delivery]. Rinsho Shinkeigaku 45: 376-379.

13. Hirsch KG, Froehler MT, Huang J, Ziai WC (2009) Occurrence o eerimesencephalic subarachnoid hemorrhage during pregnancy. Neurocrit Care 10: 339-343.

14. Verlooy J (2006) [Aneurysms of the posterior cerebral artery: comparison of a etrospective series of surgically-treated patients with endovascularly-treated patients from the literature]. Verh K Acad Geneeskd Belg 67: 91-124.

15. Honda M, Tsutsumi K, Yokoyama H, Yonekura M, Nagata I (2004) Aneurysm of the posterior cerebral artery: retrospective review of surgical treatment. Neurol Med Chir (Tokyo) 44: 164-168 